PAPER

\title{
Cueing training in the home improves gait-related mobility in Parkinson's disease: the RESCUE trial
}

\author{
A Nieuwboer, G Kwakkel, L Rochester, D Jones, E van Wegen, A M Willems, F Chavret, \\ V Hetherington, K Baker, I Lim
}

See end of article for authors' affiliations

Correspondence to: A Nieuwboer, Department of Rehabilitation Sciences, Faculty of Kinesiology and Rehabilitation Sciences, Katholieke Universiteit Leuven, Tervuursevest 101, Heverlee 3001, Belgium; alice.nieuwboer@faber. kuleuven.be

Received 15 May 2006 Revised 2 August 2006 Accepted 17 August 2006 Published Online First 22 August 2006
Objectives: Gait and mobility problems are difficult to treat in people with Parkinson's disease. The Rehabilitation in Parkinson's Disease: Strategies for Cueing (RESCUE) trial investigated the effects of a home physiotherapy programme based on rhythmical cueing on gait and gait-related activity.

Methods: A single-blind randomised crossover trial was set up, including 153 patients with Parkinson's disease aged between 41 and 80 years and in Hoehn and Yahr stage II-IV. Subjects allocated to early intervention $(n=76)$ received a 3 -week home cueing programme using a prototype cueing device, followed by 3 weeks without training. Patients allocated to late intervention $(n=77)$ underwent the same intervention and control period in reverse order. After the initial 6 weeks, both groups had a 6-week follow-up without training. Posture and gait scores (PG scores) measured at 3, 6 and 12 weeks by blinded testers were the primary outcome measure. Secondary outcomes included specific measures on gait, freezing and balance, functional activities, quality of life and carer strain.

Results: Small but significant improvements were found after intervention of $4.2 \%$ on the PG scores $(p=0.005)$. Severity of freezing was reduced by $5.5 \%$ in freezers only $(p=0.007)$. Gait speed $(p=0.005)$, step length $(p<0.001)$ and timed balance tests $(p=0.003)$ improved in the full cohort. Other than a greater confidence to carry out functional activities (Falls Efficacy Scale, $p=0.04$ ), no carry-over effects were observed in functional and quality of life domains. Effects of intervention had reduced considerably at 6-week follow-up.

Conclusions: Cueing training in the home has specific effects on gait, freezing and balance. The decline in effectiveness of intervention effects underscores the need for permanent cueing devices and follow-up treatment. Cueing training may be a useful therapeutic adjunct to the overall management of gait disturbance in Parkinson's disease.
1 n neurological patients, Parkinson's disease is the most common disorder leading to gait disturbance and falls. ${ }^{1}$ Despite advances in pharmacological treatments and surgical techniques, gait and balance deficits still persist and are associated with loss of independence, immobility and high cost for healthcare systems. ${ }^{2}$ Therefore, the development of rehabilitation approaches that work in conjunction with current treatment is important to manage these problems.

Recent systematic reviews concluded that evidence available was insufficient to support or refute the efficacy of physiotherapy in Parkinson's disease or to support the use of one form of physiotherapy over another. ${ }^{3}{ }^{4}$ Some studies had methodological problems. However, reviewers did comment that the efficacy of physiotherapy was improved by the addition of cueing techniques. Cueing is defined as using external temporal or spatial stimuli to facilitate movement (gait) initiation and continuation. Recent reviews on cueing suggest that it can have an immediate and powerful effect on gait performance in people with Parkinson's disease, indicating improvements in walking speed, step length and step frequency. ${ }^{6}$ The influence of cueing has mainly been studied in single-session experiments in laboratory settings. ${ }^{7-11}$ Results show a short-term correction of gait and gait initiation, and suggest that carry-over to uncued performance and its generalisation to activities of daily living (ADL) is limited. Using cues in a therapeutic setting is more complex, as the "modality" of cue delivery (visual, auditory or somatosensory) and the cue "parameter" selected for movement correction (frequency or size of step) have to be adapted to the needs of the patient. Apart from two limited studies on the retention effects of cues, to date no work has evaluated the clinical application and prolonged training effects of cues in the home to improve walking in a functional context. $^{12}{ }^{13}$ Furthermore, improved mobility with cues may have an adverse effect by distracting attention, increasing the risk of falling. ${ }^{14} 15$

The primary objective of this study was to investigate the efficacy of a home-based cueing programme on parameters of gait, gait-related activity and health-related quality of life in people with Parkinson's disease. We hypothesised that a 3week period of home-based cueing training would result in measurable improvements of selected gait parameters immediately after treatment, but that these effects might decrease after 6 weeks without cueing.

\section{METHODS}

\section{Study population}

We recruited 153 patients with idiopathic Parkinson's disease from three European centres: Northumbria University, Newcastle upon Tyne (UK); Katholieke Universiteit Leuven, Leuven (Belgium); and the Vrije Universiteit Medical Centre Amsterdam, Amsterdam (The Netherlands). The study was approved by the ethics committee of each participating centre. All patients gave informed written consent to the study. Eligibility criteria were: showing mild to severe gait disturbance with score $>1$ on the Unified Parkinson's Disease Rating Scale

Abbreviations: $A D L$, activities of daily living; ICC, intraclass correlation coefficient; PG score, posture and gait score; RESCUE, Rehabilitation in Parkinson's Disease: Strategies for Cueing; UPDRS, Unified Parkinson's Disease Rating Scale 
(UPDRS; item 29) ${ }^{16}$; diagnosis of idiopathic Parkinson's disease (defined by the UK Brain Bank Criteria) ${ }^{17}$; stable drug usage; Hoehn and Yahr stage II-IV; and age 18-80 years. Patients were excluded if they had undergone deep brain stimulation or other stereotactic neurosurgery; had cognitive impairment (Mini Mental State Examination Scores $<24)^{18}$; had disorders interfering with participation in cueing training, including neurological (stroke, multiple sclerosis, tumour), cardiopulmonary (chronic obstructive disorders, angina pectoris) and orthopaedic (osteoarthritis, rheumatoid arthritis and back pain) conditions; had unpredictable and longlasting off periods (score 1 on item 37 and score $>2$ on item 39 of the UPDRS) ${ }^{16}$; and had participated in a physiotherapy programme 2 months before starting the trial.

\section{Design and procedures}

The present study was a single-blind, randomised, clinical trial with a crossover design with no wash-out period. The choice of design was based on previous evidence of the short-lasting effects of cueing, ${ }^{56}$ the advantage of providing treatment for all patients, and increasing the statistical power within the constraints of research funding. In each centre, patients were randomly allocated in permuted blocks of six to an early or late intervention group by an independent investigator not involved in data analysis. Allocation was concealed by the use of opaque sealed envelopes. The early group received a cueing programme delivered in nine treatment sessions for 30 min over 3 weeks, immediately followed by 3 weeks in which no training was received. Subjects in the late group were put on a 3-week waiting list immediately followed by 3 weeks of cueing training. Both treatment arms underwent a follow-up period of 6 weeks without training. Medical treatment continued unchanged throughout the study. Before the Rehabilitation in Parkinson's Disease: Strategies for Cueing (RESCUE) trial, therapists and testers underwent separate training sessions lasting a full week to standardise the procedures in all centres.

\section{Intervention}

Cueing training was delivered in the home by one therapist at each location. A prototype cueing device, specifically developed for the study, provided three rhythmical cueing modalities: (1) auditory (a beep delivered through an earpiece); (2) visual (light flashes delivered through a light-emitting diode attached to a pair of glasses); and (3) somatosensory (pulsed vibrations delivered by a miniature cylinder worn under a wristband). Patients tried all cueing modalities in the first week, but trained with their preferred modality. By correcting the temporal aspects of gait, cueing training aimed to improve step length and walking speed, prevent freezing episodes and improve balance.

Cued practice was applied during a variety of tasks and environmental situations and consisted of the following components: gait initiation and termination, ${ }^{10}{ }^{11}$ heel strike and push-off, sideways and backwards stepping, walking while dual tasking, ${ }^{19}$ and walking over various surfaces and long distances. ${ }^{8}$ For "freezers", cues were applied to facilitate continuation of gait during turns and manoeuvres in tight places and doorways. On the basis of previous experiments undertaken by the RESCUE consortium ${ }^{19-22}$ and the literature, ${ }^{56}$ evidence-based cueing guidelines were drawn up, specifying the cueing parameters and instructions for different profiles of patients (available on CD-Rom at http://www.rescueproject.org/ ). Cues were generally delivered at patients' preferred frequency (determined for indoor and outdoor environments) and adjusted to increase step length and walking speed, depending on the aims of therapy. In case patients had freezing, cueing frequency was started at the preferred rhythm and adjusted to lower rhythms to avoid hastened stepping if needed. ${ }^{22}$ Patients were mainly instructed to match their heel strike with the cueing rhythm and keep on stepping through turns or during other manoeuvres. Specific instructions to maintain or extend step length or heel strike with every cue were added if and when required. Therapists recorded the content and amount of therapy in a diary in 15-min units.

\section{Outcome measures}

As this study wanted to measure the training effects and not the immediate effects of cueing, outcome measures were tested without the cueing device. Most outcomes described below were tested for reliability and validity at home before the trial by three testers and during two consecutive visits. Part of this study, including the full method, was published elsewhere. ${ }^{23}$ As the RESCUE trial included repeated measures performed by the same tester, the intraclass correlation coefficients (ICC) for within raters of each outcome are described as below.

1. The primary outcome measure was the posture and gait (PG) score, a composite score of gait and balance UPDRS items (13-15 and 29-30), reflecting the main aims of cueing training $($ ICC $=0.79)$.

2. To explore the specific effects of cueing training, secondary outcome measures consisted of the following:

a. Gait and balance measures: 10-m test of walking at the person's preferred walking speed, using a stopwatch to calculate gait speed $(\mathrm{m} / \mathrm{s}$; ICC $=0.81)$, step length $(\mathrm{m})$ and step frequency (steps/min); functional reach ${ }^{24}(\mathrm{ICC}=0.74)$; timed single leg and tandem stance until subjects reached a maximum of $30 \mathrm{~s}$; Freezing of Gait Questionnaire ${ }^{25}$ $($ ICC $=0.84) ;$ and Timed Get Up and Go Test ${ }^{26}$ $($ ICC $=0.88)$. Timed walking and Get Up and Go tests were standardised for each patient's home.

b. Activity measures: Nottingham Extended Activities of Daily Living Index ${ }^{27}($ ICC $=0.93)$ and Falls Efficacy Scale ${ }^{28}$ $(\mathrm{ICC}=0.88)$.

c. Participation measures: Parkinson's Disease Questionnaire$39^{29}($ ICC $=0.79)$ and Carer Strain Index ${ }^{30}($ ICC $=0.85)$.

A falls diary was left in the patient's home during the trial period to indicate the number of falls as a measure of possible adverse cueing effects. A dichotomised score was derived ( falls $=1$ or no falls $=0$ ) at crossover and at the end of therapy from the recorded number of falls during the previous 3-week periods and at follow-up for the previous 6 weeks. At test 1 , the dichotomised score was calculated from the number of falls during the previous 3 months.

Descriptive measures included the Mini Mental State Examination, ${ }^{13}$ the Brixton Test ${ }^{31}$ for executive function, and the Hospital Anxiety and Depression Scale. ${ }^{32}$

\section{Assessment protocol}

All outcome measures were assessed immediately before randomisation (test 1 ) and at 3 (test 2), 6 (test 3), and 12 weeks (test 4 ). One trained tester at each centre, not involved in training and blinded to group allocation, performed all assessments in the patient's home. Each patient was assessed at the same time of day in the on phase, approximately $\mathrm{l} \mathrm{h}$ after drug intake, to control for variations due to the drug cycle. The order of tests was randomised for each patient. Testers verified that patients had taken their drugs, and the efficacy of drugs was checked at each assessment.

\section{Statistical analysis}

Power analysis before the trial indicated that 150 patients were required for a $10 \%$ change relative to baseline values on the PG score ( 0.6 points) with a power of $80 \%$ and a critical value of $5 \%$ for statistical significance, allowing a drop-out of $10 \%$. 
The success of blinding and randomisation procedures was explored by comparing early and late groups using Wilcoxon tests Mann-Whitney $U$ tests, $\chi^{2}$ tests and unpaired t tests.

Exploratory analysis showed carry-over effects of treatment in the control period (test 3-test 2) in the early group. Hence, intervention effects were estimated using the first three assessments (tests 1-3), with multiple linear regression models accounting for repeated measures. Where appropriate, a linear, logistic or Cox regression model was fitted for each outcome with PROC MIXED and PROC GENMOD in SAS (V.8.2). In each model the same predictors were adopted, including indicators to represent time, intervention and carry-over. A logistic regression model with generalised estimating equations evaluated the effect of intervention on the risk of falling. A Cox regression model with frailty term verified the effect of intervention on the hazard of failing the timed balance tests (inability to remain standing for a maximum of $30 \mathrm{~s}$ ), using the coxph function in Splus 2000. To explore whether cueing had an effect on movements that were not targeted by training, upper limb repetitive movement scores (UPDRS items 23-25) were analysed.

Change at follow-up was assessed by comparing the change between tests 3 and 4 using a model with two factors (time and group) fitted to the outcomes of tests $1-4$ for early and late groups taken together. For continuous outcomes, a trivariate normal distribution for error components was assumed. Data were transformed where necessary to meet normality assumptions, and baseline values were reported as medians and interquartile ranges. Two-tailed analysis was performed on all tests with a significance level of $5 \%$. Given the exploratory nature of the secondary outcomes analysis, no Bonferroni correction was applied. Intervention effects are reported as $\beta$ estimates and standard errors (SE) for the linear regression models. Odds and hazard ratios $(\mathrm{OR}$ and $\mathrm{HR} ;=\exp (\beta))$ with 95\% confidence intervals are presented for the logistic and Cox regression models.

The statistical models adopted in this study were based on the assumption that missing values occur randomly and used the remaining information even if missing values were occasionally present. Missing values occurred in $1.7 \%$ of all outcomes over the four time points and $1.3 \%$ over the three time points. As all patients received treatment and only one drop-out occurred, an "intention-to-treat" analysis was not carried out.

\section{RESULTS}

\section{Trial profile}

The trial flow chart (fig 1) shows that of 289 potential candidates, 153 patients (53\%) were eligible for inclusion into the study. Patients were mostly excluded because of insufficient impairment of gait $(n=44)$, comorbidity $(n=25)$, deep brain stimulation $(n=15)$ and an inability to commit the time needed $(n=15)$. Suitable patients were randomly allocated to the early $(n=76)$ or late intervention group $(n=77)$. One patient dropped out 3 weeks after randomisation, owing to a necessary change of drug. In total, 605 (99\%) of the planned 612 measurements were performed. Drug intake remained stable throughout the trial. Comparison between the observers' guess of allocation (early or late) and the actual patient assignment indicated that the blinding procedure was successful, as $56.1 \%(\mathrm{n}=87)$ was allocated correctly and $43.1 \%$ $(\mathrm{n}=66)$ incorrectly $\left(\chi^{2}=2.94, \mathrm{p}=0.09\right)$.

\section{Comparison of early and late intervention}

Patients received equal amounts of therapy in the early $(271.8 \mathrm{~min})$ and late groups $(270.4 \mathrm{~min}) \quad$ (t statistic $=0.27$; $\mathrm{p}=0.79$ ).

No important differences were found between the clinical profiles of the two intervention groups (table 1). Most patients had mild to moderate disease severity as $46.4 \%$ of patients $(\mathrm{n}=71)$ were in Hoehn and Yahr stage II, 41.8\% $(\mathrm{n}=64)$ in stage III and $11.8 \%(\mathrm{n}=18)$ in stage IV. Both early and late groups included similar numbers of freezers defined as having at least weekly freezing episodes $(\mathrm{n}=31,20.3 \%$ in early; and $\mathrm{n}=32,20.9 \%$ in late), as defined by a score $>1$ on item 3 of the Freezing of Gait Questionnaire. Table 2 shows the median and interquartile ranges of all outcomes at tests $1-4$. No significant group differences were found for primary and secondary outcomes at test 1 , confirming the success of the randomisation procedure. Table 2 shows that $41 \%$ in the early and 39\% in the late group reported at least one episode of falling in the 3 months before the onset of trial.

\section{Treatment effects}

Table 3 shows the estimated intervention effects corrected for time and carry-over. The primary outcome, the PG score, improved by $4.2 \%$ after intervention $(p=0.005)$. As for the secondary outcomes, gait speed $(p=0.005)$ and step length $(\mathrm{p}<0.001)$ improved by $5 \mathrm{~cm} / \mathrm{s}$ and $4 \mathrm{~cm}$, respectively. No significant change in step frequency was observed $(p=0.08)$. The Functional Reach test did not show an intervention effect $(p=0.18)$. However, the clustered tandem and one leg standing tests showed that the chance of failing these tests was decreased by $36 \%$ after intervention (HR $0.64 ; 95 \%$ confidence interval (CI) 0.48 to $0.87 ; \mathrm{p}=0.003$ ). The Timed Get Up and Go test did not show improvement after intervention. As for the severity of freezing, the Freezing of Gait Questionnaire scores were not significantly affected by cueing therapy $(p=0.25)$ after transformation. Data showed a bimodal distribution indicating a group with low scores (non-freezers) and a group with high scores (freezers). When the Freezing of Gait Questionnaire scores were re-analysed on freezers only $(\mathrm{n}=63)$, defined as having at least a weekly freezing frequency, a significant reduction of $5.5 \%$ of freezing severity was found (mean (SE) $\beta$ estimate $=-1.33$ (0.48), $\mathrm{p}=0.007$ ).

Table 3 presents intervention effects that are smaller compared with the change in median values before and after intervention for the early and late groups in table 2 as a result of the statistical control for time and carry-over effects-for example, the PG score improved from a median of 6 to 4 after treatment, suggesting a $10 \%$ rather than a $4.2 \%$ change of the scoring range. Overall, carry-over effects were only significant for step length in the early group at test $3(p=0.014)$. Time effects showed significant improvements for the PG score $(p=0.03)$, gait speed $(p=0.04)$ and the TGUG $(p=0.004)$.

For the secondary outcomes in the activity domain, improvements were found on the Falls Efficacy Scale $(p=0.03)$, indicating that patients felt more confident during gait-related activities. ADL function as measured by the Nottingham Extended Activities of Daily Living Index was not significantly altered $(p=0.07)$. The Parkinson's Disease Questionnaire-39 scores $(p=0.23)$ and the Carer Strain Index were not significantly changed after intervention $(p=0.14)$. The generalised estimating equations model showed no significant increase or decrease in the probability of falling as a result of treatment (OR $1.4 ; 95 \%$ CI 0.63 to $3.1 ; \mathrm{p}=0.4$ ). Separate analysis of the upper limb repetitive movement scores of the UPDRS (III) showed no significant treatment effect $(\beta-1.1 \quad(0.62)$, $\mathrm{p}=0.08)$. Most patients $(\mathrm{n}=95,67 \%)$ chose auditory cueing as their preferred cueing modality, whereas 57 patients $(n=58$, $33 \%$ ) favoured somatosensory cueing.

\section{Follow-up (test 4 - test 3 )}

Table 3 also shows the change at follow-up. Most intervention effects in the gait and balance domains declined significantly from 6 to 12 weeks. Secondary outcomes at activity and 


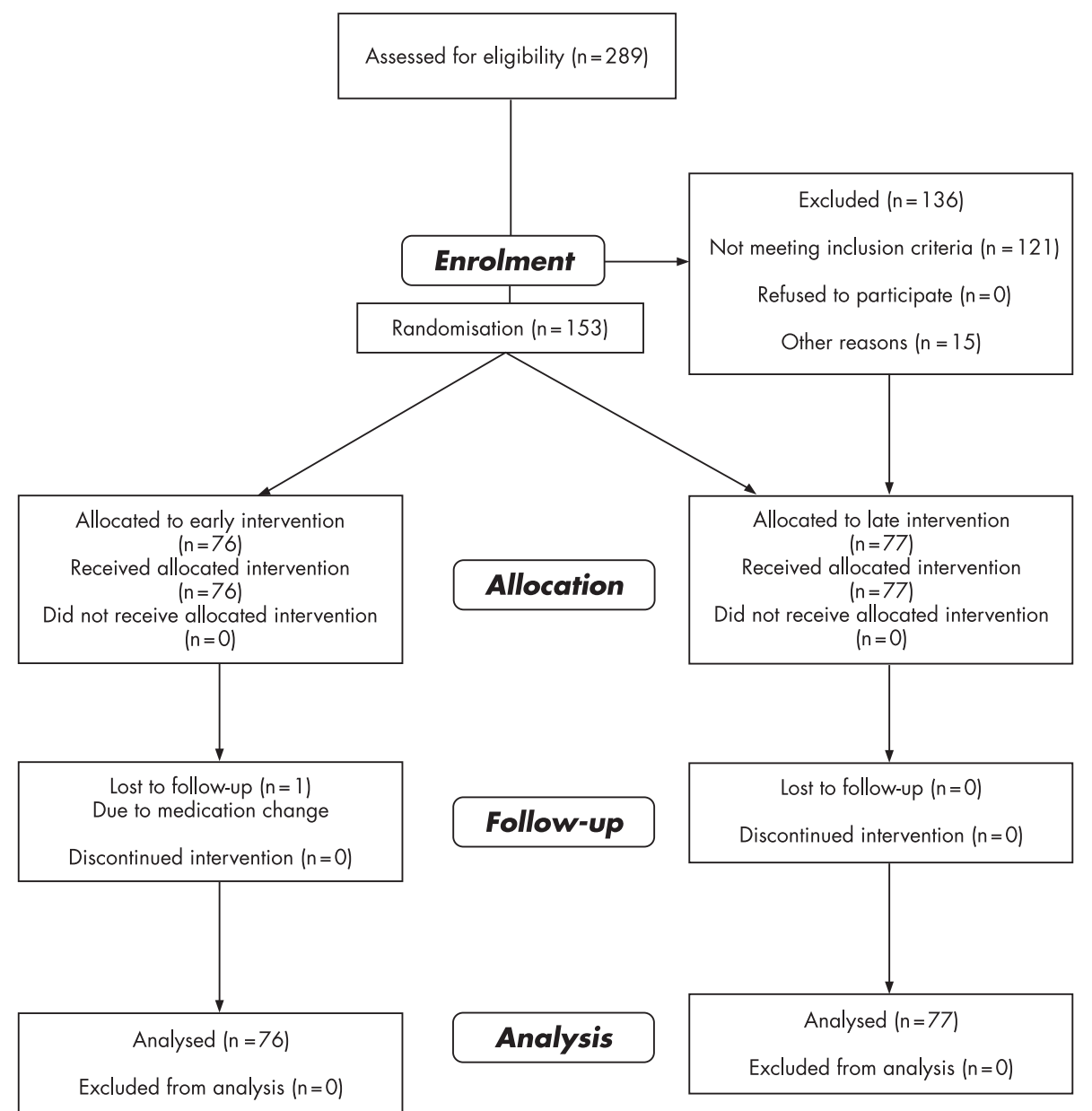

Figure 1 Trial flow chart.

Table 1 Comparison between early and late allocation groups

\begin{tabular}{llll}
\hline & $\begin{array}{l}\text { Early }(\mathbf{n}=76) \\
\text { median (IR: Q1-Q3) }\end{array}$ & $\begin{array}{l}\text { Late }(\mathbf{n}=77) \\
\text { median (IR: Q1-Q3) }\end{array}$ \\
\hline Demography & & & \\
M/F* & $48 / 28$ & $40 / 37$ & 0.16 \\
Age & $67.5(61.5-72)$ & $69(62.5-73)$ & 0.7 \\
PD characteristics & & & \\
Disease duration & $7(4-11)$ & $8(4-12)$ & 0.59 \\
H\&Y (on) & $2.5(2.5-3)$ & $3(2.5-3)$ & 0.56 \\
H\&Y II/III/IV* & $39 / 29 / 8$ & $32 / 35 / 10$ & 0.48 \\
Freezers/non- & $31 / 45$ & $32 / 45$ & 0.92 \\
freezers* & & & \\
Clinical data & & & \\
UPDRS-total (on) & $54(46-65.5)$ & $56(49-63)$ & 0.62 \\
UPDRS I (on) & $4(2-5)$ & $3(2-4)$ & 0.1 \\
UPDRS II (on) & $16(12-19.5)$ & $16(12-20)$ & 0.67 \\
UPDRS III (on) & $31(25-37)$ & $34(28-41)$ & 0.32 \\
UPDRS IV (on) & $3(1-5)$ & $2(1-5)$ & 0.43 \\
Levodopa (mg) & $500(300-700)$ & $350(200-550)$ & 0.07 \\
MMSE & $28.5(27-30)$ & $29(27-30)$ & 0.99 \\
Brixton & $4(2-6)$ & $4.0(2-6)$ & 0.45 \\
HADS anxiety & $6.5(4-10)$ & $6(4-10)$ & 0.97 \\
HADS depression & $7.5(5-10)$ & $6(4.5-9)$ & 0.45 \\
\hline
\end{tabular}

HADS, Hospital Anxiety and Depression Scale; H\&Y (on), Hoehn and Yahr stages during on; H\&Y II/III/IV, Hoehn and Yahr stages II, II, IV during on; $\mathbb{R}$, interquartile range; $M / F$, male/female; MMSE, Mini Mental State Examination; PD, Parkinson's disease; UPDRS, Unified Parkinson's Disease Rating Scale part I, II, III, IV and total score.

*Expressed as number of patients and $p$ value based on $\chi^{2}$ test. participation levels also tended to decline at test 4, a pattern that was significant for the Falls Efficacy Scale. The chance of failing the balance tests at test 4 was increased as compared with test 3 (HR 1.12; 95\% CI 0.96 to 1.32 ), but this difference was not significant $(\mathrm{p}=0.15)$. Table 2 shows that more patients reported a fall at test 4 compared with test 3 but this may reflect the fact that at test 4 a period of 6 weeks was assessed, as opposed to 3 weeks at test 3.

\section{DISCUSSION}

This is the first large-scale randomised clinical trial investigating the effects of a cueing training programme delivered at home using a multimodality cueing device. The main findings indicate that nine sessions of cueing training demonstrated considerable improvement in gait and gait-related mobility in people with Parkinson's disease, but that these effects were small and specific. The cueing method was widely accepted and well tolerated in a wide range of patients, ranging from Hoehn and Yahr stages II to IV, as evidenced by only one drop-out.

The present findings showed that a period of training with cues in the homes of people with Parkinson's disease resulted in improvement of gait immediately after intervention. We found a significant increase in walking speed and step amplitude accompanied by a tendency to reduce step frequency. This finding is in agreement with earlier work, showing that the potential to generate a more normal gait pattern can be tapped in Parkinson's disease. ${ }^{7}$ When looking at freezers 
Table 2 Medians and interquartile ranges of the outcomes in early and late intervention groups at tests 1-4

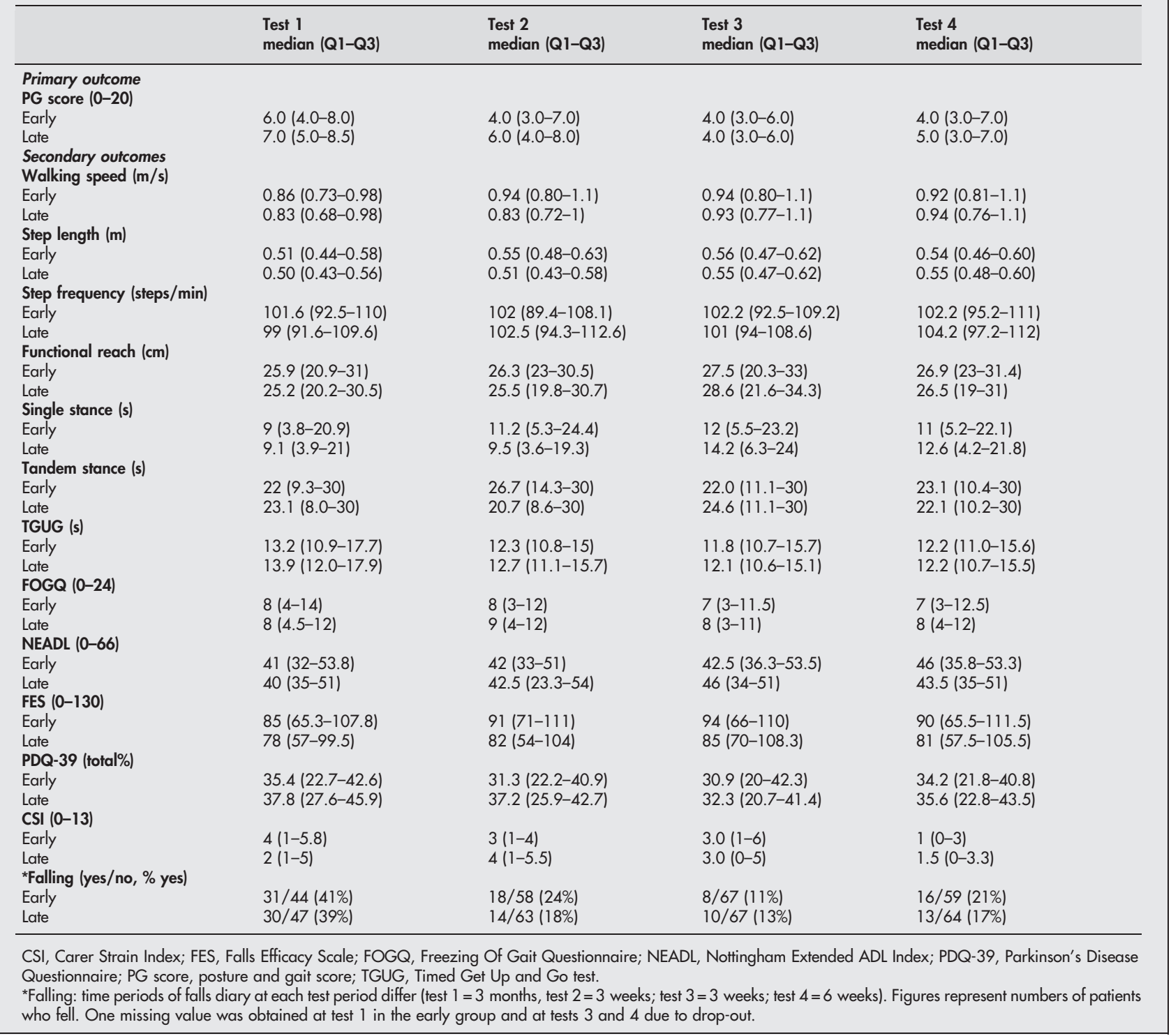

separately, a significant change on the Freezing of Gait Questionnaire after intervention was found, signifying a reduction of freezing severity. This is an important finding, as freezing is particularly resistant to drug treatment and often associated with falling. ${ }^{33}$ This result contradicts earlier work in which freezers were provided with a metronome for 1 week at home without clear benefits. ${ }^{34}$ In contrast, during the RESCUE trial, cueing therapy was provided by therapists, who used specific guidelines to set the cueing frequency to the needs of freezers, and instructed patients on how to prevent and overcome freezing in their daily environment for a 3-week period..$^{22}$

Increased rates of falls in people with Parkinson's disease are well documented and have been attributed to preserved mobility in this population. ${ }^{25}$ We were, therefore, concerned that any improvements in mobility due to therapeutic cueing could have resulted in an increased risk of falls. Our results, however, showed no evidence for this, but rather evidence of improved balance and increased confidence in the patients that they would not fall. Given the limited power to detect changes in fall rates using a fall-diary method over a short time span, the present results need cautious interpretation.
Subjects were trained for 3 weeks with cues and were evaluated without wearing the device to see if the effects were maintained. The present results showed training effects in the absence of cues, indicating that some degree of motor learning is preserved in Parkinson's disease. Whereas most studies investigated the immediate benefits of cued performance, our findings confirm the limited evidence available of improved "uncued" performance after training with cues. ${ }^{6} 812$

The effects found in this study can be considered robust and not attributable to measurement error or learning effects. All but three outcome measures had established test-re-test reliability in the home situation ${ }^{23}$ and the order of testing was randomised. To estimate the effects of intervention separately from carry-over and time effects, the statistical analysis controlled for these factors, providing a conservative estimation of treatment effects.

The fact that intervention effects were small could reflect a limitation of cueing training in the home setting. However, current effects sizes are in line with those observed in recent metaanalyses on physiotherapy in Parkinson's disease ${ }^{34}$ and in other conditions such as stroke. ${ }^{36}{ }^{37}$ The limited effects may also be explained by the relatively short training duration. Training 
Table 3 Intervention effects and change at follow-up (test 4 - test 3 )

\begin{tabular}{|c|c|c|c|c|c|}
\hline & $\begin{array}{l}\text { Intervention } \boldsymbol{\beta} \\
\text { estimate (SE) }\end{array}$ & $\begin{array}{l}\text { Change in units* } \\
\text { (\% range) } \dagger\end{array}$ & p Value & $\begin{array}{l}\text { Follow-up } \beta \text { estimate } \\
\text { (SE) test } 4 \text {-test } 3\end{array}$ & p Value \\
\hline \multicolumn{6}{|l|}{ Primary outcomes } \\
\hline PG score (0-20) & $\uparrow-0.85(0.3)$ & $4.2 \%$ & 0.005 & $\downarrow 0.582(0.14)$ & $<0.001$ \\
\hline \multicolumn{6}{|l|}{$\begin{array}{l}\text { Secondary outcomes } \\
\text { Gait and Balance }\end{array}$} \\
\hline Speed $(\mathrm{m} / \mathrm{s})$ & $\uparrow 0.05(0.02)$ & $5 \mathrm{~cm} / \mathrm{s}^{*}$ & 0.005 & $\downarrow-0.02(0.007)$ & 0.03 \\
\hline Step length $(\mathrm{m})$ & $\uparrow 0.04$ (0.009) & $4 \mathrm{~cm}^{*}$ & 0.001 & $\downarrow-0.02(0.004)$ & $<0.001$ \\
\hline Step frequency (steps/min) & $\uparrow-2.1(1.19)$ & -2.1 steps $/ \mathrm{min}^{*}$ & 0.08 & $\downarrow 1.24(0.56)$ & 0.03 \\
\hline Functional reach $(\mathrm{cm})$ & $\uparrow 1.3(0.97)$ & $1.3 \mathrm{~cm}^{*}$ & 0.18 & $\downarrow-1.08(0.46)$ & 0.02 \\
\hline Tandem stance (s) & - & - & - & - & - \\
\hline Single leg stance (s) & - & - & - & - & - \\
\hline TGUG (s) & $\uparrow-0.002(0.73)$ & $2 \mathrm{~ms}$ & 0.6 & $\downarrow 0.14(0.2)$ & 0.47 \\
\hline FOGQ (0-24) & $\uparrow-0.86(0.44)$ & $3.6 \% \dagger$ & $0.25 \ddagger$ & $\downarrow 0.8(0.21)$ & 0.001 \\
\hline \multicolumn{6}{|l|}{ Activity } \\
\hline NEADL (0-66) & $\uparrow 1.71(0.94)$ & $2.6 \% \dagger$ & 0.07 & $\downarrow-0.65(0.5)$ & 0.2 \\
\hline FES $(0-130)$ & $\uparrow 4.77(2.29)$ & $3.7 \% \dagger$ & 0.04 & $\downarrow-2.92(1.22)$ & 0.02 \\
\hline \multicolumn{6}{|l|}{ Participation } \\
\hline PDQ-39 (total\%) & $\uparrow-1.36(1.14)$ & $1.4 \%$ & 0.23 & $\downarrow 0.99(0.52)$ & 0.06 \\
\hline CSI (0-13) & $\uparrow-0.76(0.32)$ & $5.8 \% \dagger$ & $0.14 \ddagger$ & $\downarrow 0.15(0.18)$ & 0.42 \\
\hline
\end{tabular}

CSI, Carer Strain Index; FES, Falls Efficacy Scale; FOGQ, Freezing Of Gait Questionnaire; NEADL, Nottingham Extended ADL Index; PDQ-39, Parkinson's Disease Questionnaire; PG score, posture and gait score; TGUG, Timed Get Up and Go test.

$\uparrow$ Estimate represents change in direction of improvement.

$\downarrow$ Estimate represents change in direction of deterioration.

${ }^{*}$ Change expressed as measured units, positive figures represent an improvement

tChange expressed as \% of the scoring range; positive figures represent an improvement.

$\ddagger$ After transformation.

intensity rather than content was found to be a key factor in stroke research and may be equally crucial in Parkinson's disease..$^{37}$ In this study, training intensity was stipulated by the maximum number of physiotherapy sessions at home allowed for reimbursement according to existing healthcare policies. This raises the question of what the optimal duration and intensity of cueing training is and how this should be delivered over time.

What actually constitutes clinically relevant results in rehabilitation of a chronic degenerative disorder is still unclear at this point. Possibly, the improvements of gait and balance were too small to carry over to ADL and perceived quality of life. Alternatively, the narrow focus of the intervention may have led to specific effects, as we showed that repetitive upper limb movements were not affected by cueing therapy. Equally, in other fields of rehabilitation lack of generalisation is a common feature. ${ }^{36} 37$

Training effects were not sustained at 6-week follow-up as a considerable reduction in most outcomes was apparent. Similarly, Nieuwboer et $a l^{38}$ showed a considerable deterioration at 12 weeks after training with cues and Thaut $e t$ al ${ }^{39}$ found a declining slope from 4-week to 6-week follow-up. Other authors reported negligible reductions at 4-6 weeks after cueing training. ${ }^{12} 40$

The effect of placebo effects as a result of increased attention during therapy was not controlled for, which is a limitation of this study. Although the specificity of the results argues against a general effect of increased attention, effects of gait-related attention rather than cueing cannot be ruled out. Previously, it was shown that gait training with auditory cues was more effective than training without cues and no training. ${ }^{8}$

Lack of Bonferroni correction and the fact that at tests 3 and 4 testers were aware of patients having received therapy, inherent to a repeated-measures design, warrant careful interpretation of the results. However, testers were not unblinded to treatment allocation at any time point. The findings of this study cannot be generalised to people with Parkinson's disease who have significant cognitive decline and other comorbidities. Especially in the later disease stages, cues may overburden cognitive resources and increase fall risk. ${ }^{14}$ Future work should focus on determining such at-risk patients. Equally, the possibility that cues may actually reduce attentional cost in patients who are not at risk requires further investigation. Recently, we found that during cued performance of dual tasks, gait parameters improved rather than deteriorated.$^{19}$ Future studies, assessing cueing effects over a longer period of time, will be able to determine whether habituation occurs to the stimulus of the cue and verify our findings on fall risk associated with cueing over longer periods. In addition, the cost effectiveness of an extended therapeutic cueing programme possibly supplemented with a permanent cueing device needs further investigation. Although most patients preferred the auditory cueing modality $(67 \%)$, a large number of them $(33 \%)$ perceived somatosensory cueing as a well-tolerated and discrete alternative. At present, auditory cueing alone can be provided at relatively low cost using metronomes with earphones or via digital music players.

We conclude that cueing training in the home situation has a small and specific benefit for managing gait and freezing in patients with Parkinson's disease. In addition, this study has highlighted important questions on how to deliver cueing training in the most optimal way.

\section{ACKNOWLEDGEMENTS}

We thank the financial support from the European Commission Framework V funding, QLK6-2001-00120. We also thank the contribution of Professor Rowena Plant in gaining funding. Prof. ECH Wolters (MD, PhD), Dr HW Berendse (MD, PhD), CM Zijlmans (MD, PhD), Prof $\mathrm{R}$ Dom (MD, PhD), Dr D Burn (MD) and Dr R Walker (MD) are acknowledged for referral of patients. We also acknowledge help with data analysis from S Fieuws and K Desloovere, and, in particular, the patients with Parkinson's disease and their carers who participated in this study.

\section{Authors' affiliations \\ A Nieuwboer*, A M Willems, F Chavret, Katholieke Universiteit, Leuven, Belgium \\ G Kwakkel*, E van Wegen, I Lim, Vrije Universiteit Medisch Centrum, Amsterdam, The Netherlands \\ L Rochester*, D Jones*, V Hetherington, K Baker, Northumbria University, Newcastle, UK}

*These authors contributed equally to this work. 
Competing interests: The proceeds of the sale of the CD-Rom will be used to fund completion of analysis of the full RESCUE dataset. We may be involved in this further work.

\section{REFERENCES}

1 Stolze H, Klebe S, Baecker C, et al. Prevalence of gait disorders in hospitalized neurological patients. Mov Disord 2005;20:89-94.

2 Grimbergen Y, Munneke M, Bloem BR. Falls in Parkinson's disease. Review. Curr Opin Neurol 2004:17:405-15.

3 Deane KHO, Jones D, Clarke CE, et al. Physiotherapy versus placebo or no intervention in Parkinson's disease. Cochrane Database Syst Rev. 2001b: CD 002817 (3).

4 de Goede CJ, Keus SH, Kwakkel G, et al. The effects of physical therapy in Parkinson's disease: a research synthesis. Arch Phys Med Rehabil 2001;82:509-15.

5 Rubinstein T, Giladi N, Hausdorff J. The power of cueing to circumvent dopamine deficits: a review of physical therapy treatment of gait disturbances in Parkinson's disease. Mov Disord 2002;17:1148-60.

6 Lim I, van Wegen E, de Goede C, et al. Effects of external rhythmical cueing on gait in patients with Parkinson's disease: a systematic review. Clin Rehabil 2005; 19:695-713.

7 Morris ME, lansek R, Matyas TA, et al. Stride length regulation in Parkinson's disease. Normalization strategies and underlying mechanisms. Brain 1996;1 19:551-68.

8 Thaut M, Mclntosh GC, Rice RR, et al. Rhythmic auditory stimulation in gait training for Parkinson's disease patients. Mov Disord 1996;11:193-200.

9 Lewis G, Byblow WD, Walt S. Stride length regulation in Parkinson's disease: the use of extrinsic, visual cues. Brain 2000;123:2077-90.

10 Burleigh-Jacobs A, Horak FB, Nutt JG, et al. Step initiation in Parkinson's disease: influence of levodopa and external sensory triggers. Mov Disord 1997;12:206-15.

11 Dibble LE, Nicholson DE, Shultz B, et al. Sensory cueing effects on maximal speed gait initiation in persons with Parkinson's disease and healthy elders. Gait Posture 2004; 19:215-25.

12 Lehman DA, Toole T, Lofald D, et al. Training with verbal instructional cues results in near-term improvement of gait in people with Parkinson disease. J Neurol Phys Ther 2005;29:2-8.

13 Sidaway B, Anderson J, Danielson G, et al. Effects of long-term gait training using visual cues in an individual with Parkinson disease. Phys Ther 2006:86:186-94.

14 Morris $M$, lansek R, Smithson F, et al. Postural instability in Parkinson's disease: a comparison with and without a concurrent task. Gait Posture 2000;12:205-16.

15 Rochester $L$, Hetherington $V$, Jones $D$, et al. Attending to the task: interference effects of functional tasks on walking in Parkinson's disease and the role of cognition, depression, fatigue and balance. Arch Phys Med Rehabil 2004;85:1578-85.

16 Fahn S, Elton E. The Unified Parkinson's Disease Rating Scale. In: Calne D, eds. Recent developments in Parkinson's disease. New Jersey: Macmillan Healthcare Information, 1987: 153-63.

17 Hughes A, Daniel S, Kilford L, et al. Accuracy of clinical diagnosis of idiopathic Parkinson's disease: a clinico-pathological study of 100 cases. J Neurol Neurosurg Psychiatry 1992;55:181-4.

18 Folstein M, Folstein S. Mini-Mental State. A practical method for grading the cognitive state of patients for the clinician. J Psychiatr Res 1975;12:189-98.
19 Rochester L, Hetherington V Jones D, et al. The effect of external rhythmical cues (auditory and visual) on walking during a functional task in the home in people with Parkinson's disease. Arch Phys Med Rehabil 2005;86:999-1006.

20 van Wegen E, de Goede C, Lim I, et al. The effect of rhythmic somatosensory cueing on gait in patients with Parkinson's disease. J Neurol Sci 2006; [Epub ahead of print].

21 van Wegen E, Lim I, de Goede C, et al. The effects of visual rhythms and optic flow on stride patterns of patients with Parkinson's disease. Parkinsonism Relat Disord 2006;12:21-7.

22 Willems AM, Nieuwboer A, Chavret F, et al. The use of rhythmic auditory cues to influence gait in patients with Parkinsons disease, the differential effect for freezers and non-freezers, an explorative study. Disabil Rehabil 2006;28:721-8.

$23 \operatorname{Lim} \mathrm{L}$, van Wegen E, de Goede C, et al. Measuring gait and gait-related activities in Parkinson's patients own home environment: a reliability, responsiveness and feasibility study. Parkinsonism Relat Disord 2004;11:19-24.

24 Duncan $\mathrm{P}$, Weiner $\mathrm{D}$, Chandler J, et al. Functional reach: a new clinical measure of balance. J Gerontol 1990;45:M192-7.

25 Giladi N, Shabtai $H$, Simon $E$, et al. Construction of gait questionnaire for patients with Parkinsonism. Parkinsonism Relat Disord 2000;6: 165-70.

26 Morris S, Morris ME, lansek R. Reliability of measurements obtained with the timed get up \& go test in people with Parkinson's disease. Phys The 1999:81:810-18.

27 Nouri F, Lincoln N. An extended activities of daily living scale for stroke patients. Clin Rehabil 1987; 1:301-5.

28 Tinetti M, Mendes de Leon C, Doucette J, et al. Fear of falling and fall-related efficacy in relationship to functioning among community-living elders. J Geronto 1994:49:M140-7.

29 Marinus J, Ramaker C, van Hilten JJ, et al. Health related quality of life in Parkinson's disease: a systematic review of disease specific instruments. J Neurol Neurosurg Psychiatry 2002;72:241-8.

30 Robinson B. Validation of a caregiver strain index. J Gerontol 1983;38:344-8.

31 Shallice T, Burgess P. Hayling and Brixton tests (two tests of dysexecutive syndrome). England: Thames Valley Test Company, 1996.

32 Zigmund A, Snaith R. The Hospital Anxiety and Depression Scale. Acta Psychiatr Scand 1983;67:361-70.

33 Bloem B, Hausdorff J, Visser J, et al. Falls and freezing of gait in Parkinson's disease: a review of two interconnected, episodic phenomena. Mov Disord 2004; 19:871-84.

34 Cubo E, Leurgans SM, Goetz CG. Short-term and practice effects of metronome pacing in Parkinson's disease patients with gait freezing while in the 'on' state: randomized single blind evaluation. Parkinsonism Relat Disord 2004:10:507-10.

35 Ashburn A, Stack E, Pickering R, et al. Predicting fallers in a community-based sample of people with Parkinson's disease. Gerontology 2001;47:277-81.

36 Kwakkel G, van Peppen R, Wagenaar R, et al. Effects of augmented exercise therapy time after stroke: a meta-analysis. Stroke 2004;35:2529-39.

37 Van Peppen R, Kwakkel G, Wood-Dauphinee S, et al. The impact of physical therapy on functional outcomes after stroke: what's the evidence? Clin Rehabil 2004; 18:833-62.

38 Nieuwboer A, De Weerdt W, Dom R, et al. The effect of a home physiotherapy program for persons with Parkinson's disease. J Rehabil Med 2001;33:266-72.

39 Thaut MH, Mclntosh KW, Mclntosh GC, et al. Auditory rhythmicity enhances movement and speech motor control in patients with Parkinson's disease. Funct Neurol 2001;16:163-72.

40 Marchese R, Diverio M, Zucchi F, et al. The role of sensory cues in the rehabilitation of parkinsonian patients: a comparison of two physical therapy protocols. Mov Disord 2000;15:879-83. 


\section{REFERENCES}

1. Torbergsen T. Rippling muscle disease: a review. Muscle Nerve 2002;(Suppl 11):S103-7.

2. Woodman SE, Sotgia F, Galbiati F, et al. Caveolinopathies: mutations in caveolin-3 cause four distinct autosomal dominant muscle diseases. Neurology 2004;62:538-43.

3. Koul RL, Chand RP, Chacko A, et al. Severe autosomal recessive rippling muscle disease. Muscle Nerve 2001;24:1542-7.

4. Ansevin CF, Agamanolis DP. Rippling muscles and myasthenia gravis with rippling muscles. Arch Neurol 1996;53:197-9.

5. Vernino S, Auger RG, Emslie-Smith AM, et al. Myasthenia, thymoma, presynaptic antibodies, and continuum of neuromuscular hyperexcitability. Neurology 1999:53:1233-9.

6. Vernino $\mathbf{S}$, Lennon VA. Ion channel and striational antibodies define a continuum of autoimmune neuromuscular hyperexcitability. Muscle Nerve 2002;26:702-7.

7. Muller-Felber W, Ansevin CF, Ricker K, et al. Immunosuppressive treatment of rippling muscles in patients with myasthenia gravis. Neuromuscul Disord 1999;9:604-7.

8. Greenberg SA. Acquired rippling muscle disease with myasthenia gravis. Muscle Nerve 2004;29:143-6.

9. Schulte-Mattler WJ, Kley RA, Rothenfusser-Korber $\mathrm{E}$, et al. Immune-mediated rippling muscle disease. Neurology 2005:64:364-7.

10. Baker SK, Tarnopolsky MA. Sporadic rippling muscle disease unmasked by Simvastatin. Muscle Nerve 2006:34:478-81.

\section{BOOK REVIEW}

Vascular cognitive impairment in
clinical practice

Edited by L-0 Wahlund, T Erkinjuntti, S Gauthier. Published by Cambridge University Press, Cambridge, 2009, pp 241 US\$130. ISBN 978-0-521-87537-0

In 2006, the NIH convened a conference, which attempted to establish a new concept, vascular cognitive impairment (VCI). The resulting document has been published ${ }^{1}$ and now, 3 years later, several of the key participants, and others, have contributed to a new book on that topic. Edited by leading authorities and joined by several eminent experts, the book addresses clinicians dealing with demented individuals in order to distribute the concept of VCI which has not been accepted unopposed.

The term VCI is supposed to cover the whole spectrum of cognitive decline which occur as a result of vascular changes to the brain. Because these changes result from a multitude of factors (haemorrhage, ischaemia, emboli, small vessel disease, etc), VCI cannot be considered a disease, but neither is it a syndrome since there is very little in common between cognitive changes resulting from different processes (eg, leucoaraiosis and those due to a single thalamic stroke).

In addition, the vascular lesions occurring mostly in elderly subjects rarely affect an otherwise normal brain. Alzheimer pathology is very common in the elderly, and even if changes fail to satisfy arbitrary pathological criteria of Alzheimer's disease (AD), they cannot and should not be ignored. In fact, a growing body of evidence forces us to accept that most cases of dementia in the elderly are the result of combined lesions, ${ }^{2}$ and therefore the concept of (pure) VCI may be misleading. The authors of several chapters in the book acknowledge these issues, albeit in a soft voice. It seems that they try to simplify the question of the pathogenesis and manifestations of dementia in old age by resorting to a dualistic approach. Unfortunately, this entrenched position does not benefit scientific progress.

The authors do not even agree on the definition of VCI. Whereas the editors limit it to "from the earliest deficit" (p4), others ask whether VCI can be a prodrome to vascular dementia (p11). If vascular dementia is included in the spectrum of VCI, how can VCI be a prodrome to it? Still others claim that the "brain at risk stage" (ie, prior to cognitive changes) should be included (p54).

Particularly disappointing are the chapters on treatment where data from $\mathrm{AD}$ are used freely without mentioning that they do not necessarily apply to other dementia types, particularly vascular dementia. The chapter on "control of vascular risk factors" contains a well structured review of the role of dementia. However, if VCI has so many underlying pathologies, preventative treatments are hard to identify to any of its subtypes. A discussion as to why none of the anti-AD drugs has been accepted by authorities for treatment of vascular dementia is missing. Is rigid control of the risk factors useful in preventing, or at least slowing, the cognitive deterioration? This important clinical question is neglected. While there is some discussion of symptomatic therapies, this is directed at manifestations of dementia in general, perhaps because the authors believe that the clinical behavioural manifestations are similar, both in underlying mechanisms and in presentation, in VCI and in other dementia syndromes. Among atypical antipsychotic agent, the most atypical, clozapine, is not mentioned.

There are a few errors, for example, the claim that "the diagnosis of dementia can be done at the bedside using global cognitive measures (eg, Mini-Mental State Examination)", p32. Sometimes statements are made without reference (eg, "The levels of $A \beta$ may be reduced in the users of statin and Ginko biloba", p85).

While the authors of the different chapters have generally completed well the missions assigned to them, the editors and publisher could have done more. Each chapter has its own structure. Summaries, which are so important in such a comprehensive work, are missing in some chapters and replaced by "significance", "conclusions", "concluding remarks", etc, in others. The reference list after each chapter is not always arranged correctly alphabetically, and a unified reference list at the end of the book could avoid unnecessary repetitions. Sometimes unclear references are included (eg, "BNF 2006", p216).
A good index is a very important tool in a book such as this but, unfortunately, it is rather incomplete. For example, "Binswanger's disease" is mentioned in the text more frequently then four times, as suggested by the index. Abbreviations are used without spelling them out first. Table 14.1, among others, has not been proofread.

Surely, a distinguished publisher like Cambridge University Press could have done a better job.

Tel-Aviv University Medical School, Ramat Aviv 69978, Israel; amoskor@post.tau.ac.il

Provenance and peer review: Commissioned; externally peer reviewed.

J Neurol Neurosurg Psychiatry 2010;81:126. doi:10.1136/jnnp.2009.179176

\section{REFERENCES}

1. Hachinski V, ladecola C, Petersen RC, et al. Stroke 2006;37:2220-41

2. Korczyn AD. Mixed dementia - the most common type of dementia. Ann NY Acad Sci 2002;977:129-34.

\section{CORRECTIONS}

doi:10.1136/jnnp.2005.086579corr1

J D Putzke, N R Whaley, Y Baba, et al Essential tremor: predictors of disease progression in a clinical cohort. I Neurol Neurosurg Psychiatry 2006;77:1235-1237. This article was originally published with an incorrect digital object identifier (doi) of 10.1136/jnnp.2006.086579. It has been updated online with the correct doi 10.1136/jnnp.2005.086579.

doi:10.1136/jnnp.2006.095547corr1

E J Thompson. Lock and key approach to "hidden" encephalitis. J Neurol Neurosurg Psychiatry 2006;77:901. This article was originally published with an incorrect digital object identifier (doi) of 10.1136/jnnp.2005. 095547. It has been updated online with the correct doi: 10.1136/jnnp.2006.095547.

\section{doi:10.1136/jnnp.2006.097923corr1}

A Nieuwboer, G Kwakkel, L Rochester, et al Cueing training in the home improves gait-related mobility in Parkinson's disease. J Neurol Neurosurg Psychiatry 2007;78: 134-40. This paper was published Online First with an incorrect digital object identifier of 10.1136/jnnp.200X.097923. The DOI should be 10.1136/jnnp.2006.097923.

\section{doi:10.1136/jnnp.2006.0103135corr1}

J L Dornhoffer, M Mennemeier. Transcranial magnetic stimulation and tinnitus: implications for theory and practice. J Neurol Neurosurg Psychiatry 2007;78:113. This article was originally published with an incorrect digital object identifier (doi) of 10.1136/ jnnp.2006.103135. It has been updated online with the correct doi: 10.1136/jnnp. 2006.0103135 
Although it could be argued that this reduction may simply be the result of reduced life expectancy in MS patients, this is unlikely, as an age-specific Cox survival model also showed a significant reduction in the risk of cancer. ${ }^{2}$ Similarly, it is unlikely that this would represent under-reporting of cancer because patients are typically in closer contact with health practitioners than the normal population. ${ }^{2}$ Explanations could include lifestyle alterations following diagnosis, genetic factors or immunological changes due to MS. Further study of mechanisms is therefore warranted, but more immediately, the results of this metaanalysis will be of use for MS patients and their care givers.

Acknowledgements We would like to thank all members of the Ebers group for helpful discussion and frequent support.

Adam E Handel, ${ }^{1,2}$ Sreeram V Ramagopalan ${ }^{1,2}$

${ }^{1}$ Wellcome Trust Centre for Human Genetics, University of Oxford, Oxford, UK; ${ }^{2}$ Department of Clinical Neurology, University of Oxford, John Radcliffe Hospital, Oxford, UK

Correspondence to Dr Sreeram V Ramagopalan, The Wellcome Trust Centre for Human Genetics, University of Oxford, Roosevelt Drive, Oxford OX3 7BN, UK; sreeramr@well.ox.ac.uk

Competing interests None.

Patient consent Obtained.

Provenance and peer review Not commissioned; externally peer reviewed.

Received 21 September 2009

Revised 11 November 2009

Accepted 16 November 2009

Published Online First 19 August 2010

J Neurol Neurosurg Psychiatry 2010;81:1413-1414. doi:10.1136/innp.2009.195776

\section{REFERENCES}

1. Fois AF, Wotton CJ, Yeates D, et al. Cancer in patients with motor neuron disease, multiple sclerosis, and Parkinson's disease: record-linkage studies. J Neurol Neurosurg Psychiatry 2010;81:215-21.

2. Bahmanyar S, Montgomery SM, Hillert J, et al. Cancer risk among patients with multiple sclerosis and their parents. Neurology 2009; 72:1170-7

3. Lebrun C, Debouverie M, Vermersch $P$, et al. Cancer risk and impact of disease-modifying treatments in patients with multiple sclerosis. Mult Scler 2008;14:399-405.

4. Nielsen NM, Rostgaard K, Rasmussen S, et al. Cancer risk among patients with multiple sclerosis: a population-based register study. Int J Cancer 2006;118:979-84.

5. Midgard R, Glattre E, Gronning M, et al. Multiple sclerosis and cancer in Norway. A retrospective cohort study. Acta Neurol Scand 1996;

93:411-15.

\section{CORRECTIONS}

doi:10.1136/jnnp.2008.169029corr1

J Pretnar-Oblak, M Zaletel, T M Hajnšek, et al. Isolated bulbar paralysis in a patient with medullar tau pathology: a case report (J Neurol Neurosurg Psychiatry 2010;81: 847-849). The authors misplaced the label number (9) in Figure 1 of this paper and therefore this area indicated does not represent the nucleus ambiguous. The reprinted version of Figure 1 represents the correct area for nucleus ambiguous.

The authors would also like to explain further the labels in figure $2 \mathrm{C}$ and $2 \mathrm{D} .2 \mathrm{C}$ : Extensive tau pathology in DNVN composed of numerous neuropil threads and tau-positive neurons (arrows). 2D: Tau pathology of few neuropil threads in the SN (arrows).

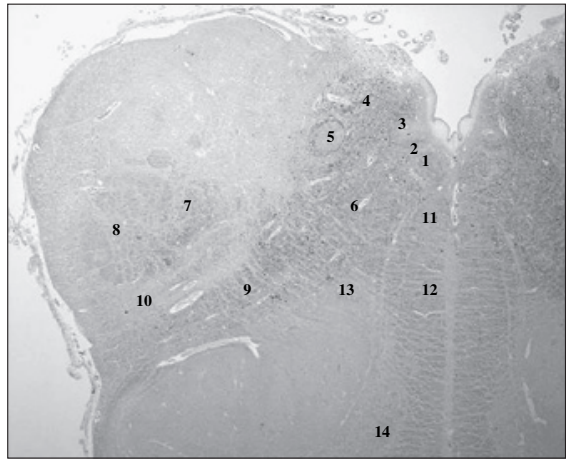

Figure 1 The corrected version with previously misplaced number 9 .

doi:10.1136/jnnp.2006.089540

Jes Olesen, Mary G Baker, Tamas Freund, et al. Consensus document on European brain research (J Neurol Neurosurg Psychiatry doi:10.1136/jnnp.2006.095547

2006;77:i1-i49). This paper was published in the journal without a doi. The doi is $10.1136 /$ jnnp.2006.089540.

E J Thompson. Lock and key approach to "hidden" encephalitis (J Neurol Neurosurg Psychiatry 2006;77:901). This paper was published in the journal with an incorrect doi of 10.1136/jnnp.2005.095547. The correct doi is 10.1136/jnnp.2006.095547.

doi:10.1136/jnnp.2006.097923

Alice Nieuwboer, Gert Kwakkel, Lynn Rochester, et al. Cueing training in the home improves gait-related mobility in Parkinson's disease: The RESCUE-trial (J Neurol Neurosurg Psychiatry 2007;78:134-140. This paper was published in the journal with an incorrect doi of doi:10.1136/ jnnp.2005.097923. The correct doi is 10.1136/jnnp.2006.097923.

doi:10.1136/jnnp.2006.103135

J L Dornhoffer, M Mennemeier. Transcranial magnetic stimulation and tinnitus: implications for theory and practice ( N Neurol Neurosurg Psychiatry 2007;78:113). This paper was published in the journal with an incorrect doi of 10.1136/jnnp.2006.0103135. The correct doi is $10.1136 /$ jnnp.2006.103135.

doi:10.1136/jnnp.2008.146548

S T Andersen, J Vissing. Carbohydrateand protein-rich diets in McArdle disease: effects on exercise capacity ( N Neurol Neurosurg Psychiatry 2008;79:1359-1363). This article was published in the journal with an incorrect doi of 10.1136/ adc.2008.146548. The correct doi is 10.1136/jnnp.2008.146548. 